Anaesthesist 2013 $\cdot 62: 341-342$

DOI 10.1007/s00101-013-2165-y

Online publiziert: 8. Mai 2013

(c) Springer-Verlag Berlin Heidelberg 2013

\section{T. Weig}

Klinik für Anaesthesiologie, Klinikum der Universität München

\section{Peripartale Kardiomyopathie}

Schwangerschaft und Geburt eines Kindes sind herausragende Erlebnisse für die Frau und die Familie. Eine im Rahmen der Schwangerschaft oder der postpartalen Phase erforderliche Intensivtherapie stellt für die Betroffenen ein dramatisches Ereignis dar. Aber auch für das Behandlungsteam bedeutet dies eine besondere fachliche und emotionale Herausforderung. Dies gilt im besonderen Maß, wenn die Intensivtherapie ante partum erforderlich wird.

Aufgrund der Zunahme des mütterlichen Alters und des Bestrebens, den Kinderwunsch chronisch schwer erkrankter Frauen zu erfüllen, werden Anästhesisten und Intensivmediziner als Teil des multidisziplinären Behandlungsteams zunehmend mit komplexen Konstellationen konfrontiert. Diese Patientinnen bedürfen der sorgfältigen Betreuung während der gesamten Schwangerschaft, insbesondere aber in der peripartalen Phase.

Daten aus Südengland zeigen, dass die häufigsten Ursachen für die Notwendigkeit einer intensivmedizinischen Betreuung peripartaler Patientinnen schwangerschaftsassoziierte hypertensive Erkrankungen (39,5\%) und postpartale Hämorrhagien $(33,3 \%)$ sind [1]. Beide Krankheitsbilder sind gut bekannt, klinisch meist eindeutig zu diagnostizieren und ziehen klare therapeutische Konsequenzen nach sich.

Die Autoren des aktuellen Leitthemas in dieser Ausgabe von Der Anaesthesist nehmen sich des seltenen, aber wichtigen Krankheitsbilds der peripartalen Kardio- myopathie an. Sie beschreiben detailliert, wie schwierig das frühzeitige Erkennen dieser oft dramatisch verlaufenden Erkrankung ist, ganz im Gegensatz zu den oben genannten „häufigen Diagnosen“. Gerade wir als Vertreter des Querschnittfachs Anästhesie mit unserer Expertise in der Intensiv- und Notfallmedizin sind gefragt, die Differenzialdiagnose „peripartale Kardiomyopathie" in die Waagschale zu werfen, wenn uns die Kollegen der Gynäkologie eine Patientin mit vielleicht nur diskreten Zeichen einer Herzinsuffizienz vorstellen. Nach der Lektüre des Beitrags sind die Sinne hierfür geschärft. Nicht nur in Bezug auf Erkennen und Diagnostik geben die Autoren dem Leser einen guten Überblick, sondern beschreiben anhand der ausführlichen Aufarbeitung der Literatur auch die therapeutischen Optionen, begonnen von der präoperativen Planung über die perioperative Strategie bis hin zur Langzeittherapie.

Der Blick in die Autorenliste macht dem Leser die eminente Bedeutung einer interdisziplinären Zusammenarbeit einer Vielzahl von Fachgebieten deutlich. Wir nehmen dabei zusätzlich zu der Aufgabe der akuten Stabilisierung der Patientin auch die Schlüsselrolle als Koordinatoren der einzelnen Fachgebiete ein. Der Appell der Autoren, Patientinnen mit vermuteter oder diagnostizierter peripartaler Kardiomyopathie frühzeitig an ein überregionales Zentrum mit allen Therapieoptionen bis hin zur maschinellen Herzunterstützung zu verlegen, kann auch aus eigener Erfahrung nur unterstützt werden [2]. 
Anhand der das Krankheitsbild facettenreich illustrierenden Fallvignette diskutieren die Autoren kritisch die bei ihrer Patientin durchgeführte Therapie der peripartalen Kardiomyopathie. Insbesondere analysieren sie die Therapie auch unter den Aspekten „Evidenz“ und „off label use".

Die Cochrane Collaboration konnte kürzlich lediglich eine einzige klinische (Pilot-)Studie zur Therapie der peripartalen Kardiomyopathie identifizieren, die den Kriterien der evidenzbasierten Medizin gerecht wurde [3, 4]. Basierend auf den positiven Ergebnissen dieser Pilotstudie führen Hilfiker-Kleiner et al. von der Medizinischen Hochschule Hannover derzeit eine Multizenterstudie zur Bromocriptintherapie bei der peripartalen Kardiomyopathie durch (ClinicalTrials.gov Identifier: NCT00998556). An der University of Pittsburgh wurden $100 \mathrm{~Pa}$ tientinnen in eine Observationsstudie aufgenommen, um die Ursachen der Erkrankung genauer zu identifizieren (ClinicalTrials.gov Identifier: NCT01085955). Eine erste Auswertung der Ergebnisse wird noch 2013 erwartet. Es tut sich also etwas im spannenden Gebiet der peripartalen Kardiomyopathie!
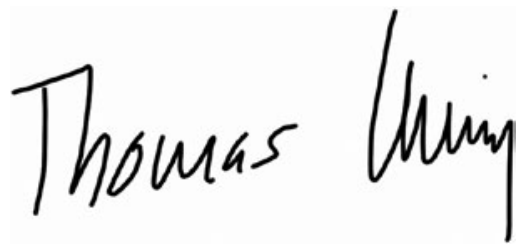

Thomas Weig

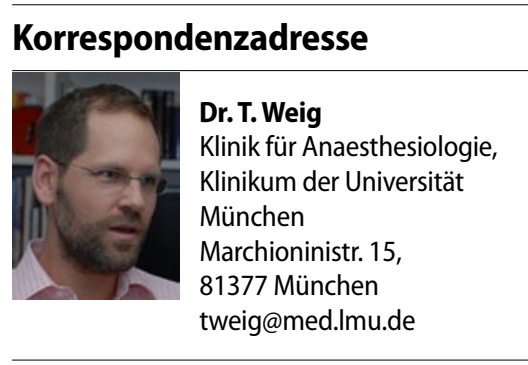

Interessenkonflikt. Der korrespondierende Autor gibt an, dass kein Interessenkonflikt besteht.

\section{Literatur}

1. Hazelgrove JF, Price C, Pappachan VJ, Smith GB (2001) Multicenter study of obstetric admissions to 14 intensive care units in southern England. Crit Care Med 29:770-775

2. Weig T, Dolch ME, Frey L et al (2011) Delayed intracardial shunting and hypoxemia after massive pulmonary embolism in a patient with a biventricular assist device. J Cardiothorac Surg 6:133

3. Carlin AJ, Alfirevic Z, Gyte GM (2010) Interventions for treating peripartum cardiomyopathy to improve outcomes for women and babies. Cochrane Database Syst Rev:CD008589

4. Sliwa K, Blauwet L, Tibazarwa K et al (2010) Evaluation of bromocriptine in the treatment of acute severe peripartum cardiomyopathy: a proof-ofconcept pilot study. Circulation 121:1465-1473

\section{AGNN-Preis für Notfallmedizin}

Die Arbeitsgemeinschaft in Norddeutschland tätiger Notärzte e.V. (AGNN) verleiht alle zwei Jahre den von ihr gestifteten „AGNN-Preis für Notfallmedizin" für herausragende Projekte und / oder wissenschaftliche Arbeiten auf dem Gebiet der Notfallmedizin.

Im Rahmen des 9. Notfallsymposiums der Arbeitsgemeinschaft in Norddeutschland tätiger Notärzte e.V. (AGNN) in Lübeck-Travemünde wurde am 20. April 2013 der „AGNN-Preis für Notfallmedizin“ verliehen.

Träger des mit $€ 500$ dotierten Preises ist Dr. Roman Lukas aus der Arbeitsgruppe Forschung in der Notfallmedizin (Klinik für Anästhesiologie, operative Intensivmedizin und Schmerztherapie, Universitätsklinikum Münster und Berufffeuerwehr Münster).

Dr. Lukas wurde für seine Arbeit "Qualitätsmanagement der Thoraxkompression und das Primärüberleben bei der Reanimation: Eine registerbasierte matched-pair Analyse" ausgezeichnet.

In der Arbeit konnten die Münsteraner Forscher um Dr. Lukas anhand einer matched-pair Analyse mit Hilfe des Deutschen Reanimationsregisters zeigen, dass ein Qualitätsmanagement für die Thoraxkompression bei der präklinischen Reanimation zu signifikant höheren Überlebensraten führt, als mittels Vorhersagewert (RACAScore) prognostiziert.

Hierzu wurden alle nicht-traumatischen Reanimationen in Münster aus den Jahren 2007 bis 2011 mit Hilfe des Deutschen Reanimationsregisters analysiert.

Die Arbeit wurde Oktober 2012 im Journal „Resuscitation“ veröffentlicht: Lukas RP et al., Chest compression quality management and return of spontaneous circulation: A matched-pair registry study. Resuscitation. 2012 Oct;83(10):1212-1218.

Der AGNN-Preis wird alle zwei Jahre verliehen und würdigt herausragende Projekte und / oder wissenschaftliche Arbeiten auf dem Gebiet der Notfallmedizin.

Quelle: AGNN 